\title{
5. LONG-TERM VARIATIONS IN METEOR RADAR RATES, METEOR HEIGHTS AND RADAR-ECHO AMPLITUDES
}

\author{
B. A. LINDBLAD \\ (Lund Observatory, Sweden)
}

\begin{abstract}
A BST RACT
An analysis of meteor radar rates, meteor end-point heights and radar-echo amplitudes observed over the period 1953-66 indicates a long-term variation in these quantities with highest values being recorded in 1963 near solar minimum. The phenomenon is explained in terms of a solar-controlled variation in air density at the $90-$ to $110-\mathrm{km}$ level, with highest densities occurring in 1963 . This provides an explanation for the anomalous increase in meteor radar rates observed in 1963. The analysis indicates that air density at the meteor burn-out level varies inversely as solar flux. The density variation is thus opposite in phase to that at higher altitudes as deduced from satellite drag measurements.
\end{abstract}

\section{Introduction}

Several investigators have attempted to find statistical correlations between meteor phenomena and indices of solar activity. Bumba (1949) correlates the occurrence rates of various meteor and fireball phenomena with the position in the solar cycle. Lowest rates of sporadic and shower meteors were observed to occur about 1 year before a solar maximum and highest rates about 5-6 years later. Bumba (1955) also found a correlation between the mean geocentric velocity of sporadic meteors and solar activity.

The data sample used in the above correlation studies was very heterogeneous, and it is difficult to assess the statistical significance of these investigations. It is evident that the statistical correlations obtained need to be explained in terms of physical processes occurring in the upper atmosphere. Until recently, our understanding of these processes was very incomplete.

The introduction of radio techniques for the investigation of meteors enabled longterm systematic meteor studies, and several research groups are currently engaged in extensive observational programs. In 1963 radar research groups in New Zealand, Canada, and Sweden all noted an unexpected increase in the occurrence rate of meteor radar echoes. Subsequent studies by Ellyett and Keay (1964), McIntosh and Millman (1964), McIntosh (1967) and Lindblad (1967a) showed that the echo rate in 1963 was a factor of $1 \cdot 5-2$ higher than in previous years. From the above studies it was evident that the 1963 increase in meteor echo rates was a world-wide phenom-

Kresák and Millman (eds.), Physics and Dynamics of Meteors, 50-62. C. I.A.U. 
enon. An instrumental origin was thus excluded. McIntosh (1967) suggested an increase in atmospheric density at the meteor burn-out level as a possible explanation. A density increase in a localized height region between 90 and $110 \mathrm{~km}$ will raise the detection threshold of a meteor radar and thus give rise to an apparent increase in the meteor rate.

The investigation of Lindblad (1967a) indicated that meteor rates (and heights) had varied periodically from 1953 to 1965, apparently as a function of position within the solar cycle. In this study, hereinafter referred to as Paper I, the observed changes in meteor rates and heights were interpreted in terms of a solar-controlled variation in the atmospheric density gradient at the meteor burn-out level. It was tentatively estimated that neutral density in the $90-$ to $110-\mathrm{km}$ region of the Earth's atmosphere may vary over the solar cycle by a factor of between 1.5 to 1.7 , with maximum density during the solar minimum phase. The 1963 increase in radar echo rates is thus explained as the result of exceptionally high densities in the $90-$ to $110-\mathrm{km}$ region concurrent with the minimum phase of solar activity.

In a further study Lindblad (1967b) analysed rocket-determined atmospheric densities. For the 90 - to $110-\mathrm{km}$ region, density data derived from falling-sphere experiments in 1961-66 confirmed the solar cycle dependence over the solar minimum phase. In confirmation of the meteor results highest atmospheric densities in this region were recorded in 1963.

\section{Present Investigation}

In the present study additional experimental data are presented on the long-term variation of meteor parameters. The data were collected in 1953-66 as part of a cooperative program of meteor research carried out by the Lund and Onsala observatories in Sweden. The data available for analysis were observations by radar and visual techniques of Perseid and sporadic meteors. Observational and reductional techniques of this program as well as basic parameters of the radar equipment have been reported earlier (Lindblad, 1963, 1967a).

The main emphasis of our project is directed to a long-term study of the Perseid meteor shower. Radar observations are therefore carried out mainly during August, with only occasional check runs at other times. The standard observational program during the August epoch consists of a night-time recording period between $22 \mathrm{hrs}$ and $04 \mathrm{hrs}$ MET, and a day-time run between $08 \mathrm{hrs}$ and $14 \mathrm{hrs}$ MET. The night-time program has been operated regularly since 1953; the day-time program since 1960.

The night-time and day-time radar programs are symmetrical with respect to the motion of the Perseid radiant, and the elevation settings of the radar beam are such that the mean collecting area of the beam is the same during both runs. Mean echo rates of the two programs should thus be directly comparable. 


\section{Radar Echo Rates}

\section{A. PRESENTATION OF DATA}

The total number of echoes recorded during an operational run, divided by the total observing time in hours, defines the hourly rate. Total night-time hourly rates for the August epoch, as recorded in 1953-65, are shown in Figure 1A (filled circles). Total day-time hourly rates for the 1960-65 period are shown in Figure 1B (filled circles). The August observations were supplemented on a semi-regular basis by a check run 2-4 weeks after the Perseid epoch. Total hourly rates obtained during the check runs are given in Figure 1 (open circles).

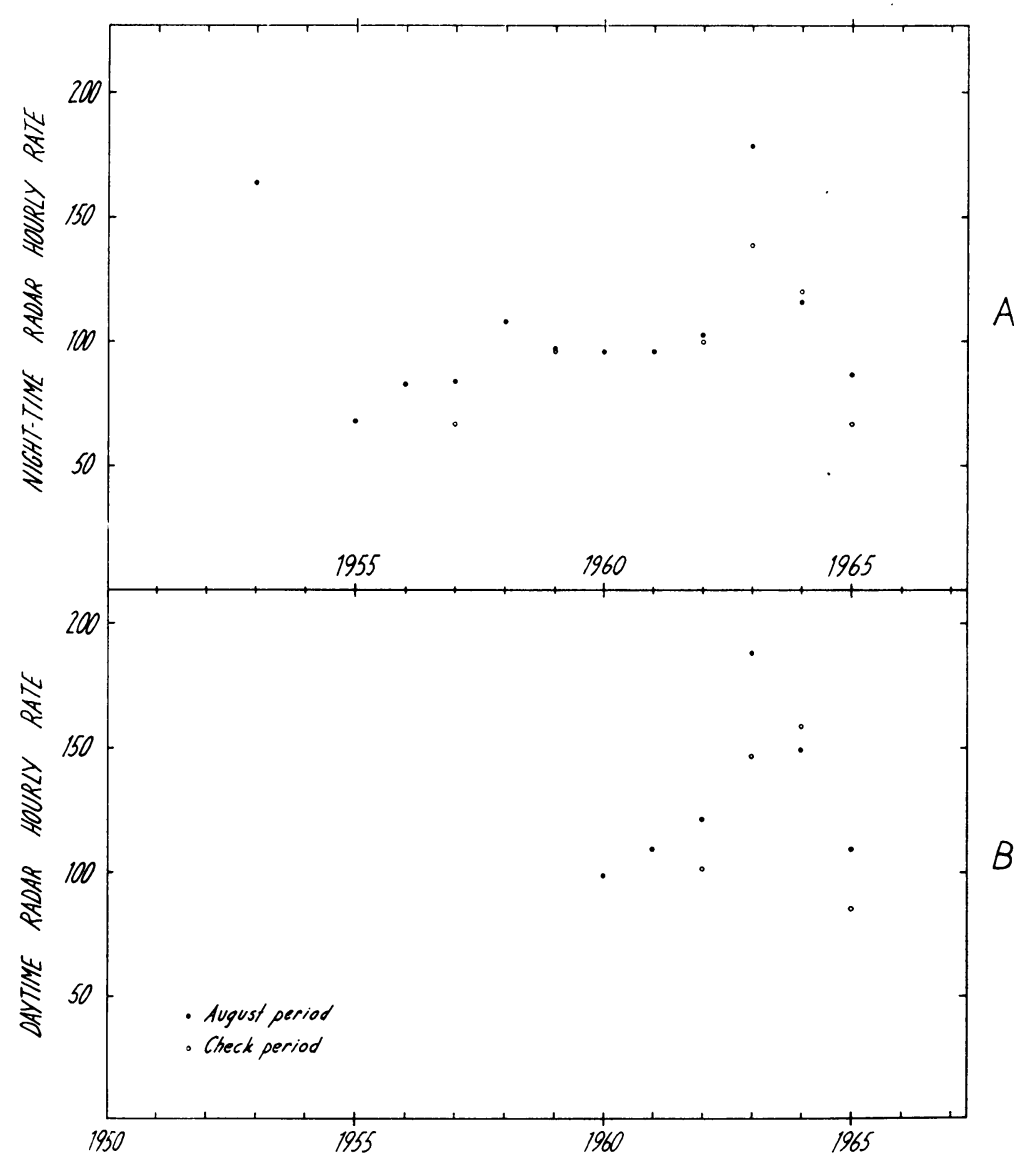

FIG. 1. Night-time and day-time radar hourly rates $1953-65$, all echoes. 


\section{B. DISCUSSION OF DATA}

The night-time radar rates shown in Figure $1 \mathrm{~A}$ have been reported previously (Paper I). From the data presented it was concluded that meteor echo rates exhibit a secular or long-term variation, which is related to solar activity. It was observed that lowest echo rates were recorded during - or slightly before - the solar maximum phase and highest rates during the solar minimum phase.

The operational run in August includes the period of maximum activity of the Perseid shower, but the length of the run is such that the observed total rates may be considered a measure of sporadic meteor activity. Figure 1 shows that total echo rates for the check periods closely follow the same trend as the August rates. It is therefore concluded that the observed time variation in echo rates is representative of meteor echo rates in general.

Day-time total echo rates are shown in Figure 1B. These data have not been reported previously. The day-time recordings are subject to more severe radio interference than the night-time recordings, and it may be argued that a spurious variation in day-time echo counts could be introduced by a long-term, solar-controlled variation in the level of radio interference. Figure 1 shows that the day-time and night-time echo rates exhibit similar temporal variations. It is therefore concluded that the recorded longterm variations in the day-time data are significant.

\section{ECHO RATES IN DIFFERENT DURATION GROUPS}

In a study of the 1963 anomalous increase McIntosh and Millman (1964) analysed the occurrence rate of echoes of duration $>8 \mathrm{sec}$. In this long-duration group no significant increase in the echo rate for 1963 was observed. McIntosh and Millman therefore concluded that the 1963 increase was restricted to short-duration echoes.

In view of the different scattering properties of overdense and underdense meteor trails, it is of interest to investigate in our data if the observed temporal variations in echo rates occur amongst both types of echo signals. For the purpose of this study meteor echo signals were classified into three duration groups: echoes longer than $1 \mathrm{sec}$; echoes of duration $1.0-0.5 \mathrm{sec}$ and echoes shorter than $0.5 \mathrm{sec}$. The first two groups correspond mainly to overdense meteor trails, while the third group corresponds to the underdense type of meteor trail.

Separate hourly rates for the three duration groups are given in Figure 2. The diagram covers the 1953-65 August period and both night-time and day-time data are included. Figure 2 shows that all data samples exhibited similar temporal variations with the highest rates in 1963-64. The maximum in echo rates is, however, somewhat more pronounced in the underdense echo group.

In Figure 2 a datum point for 1953 is only given for the group of shortest duration, since the echo classification used for the 1953 data only distinguished between echoes 


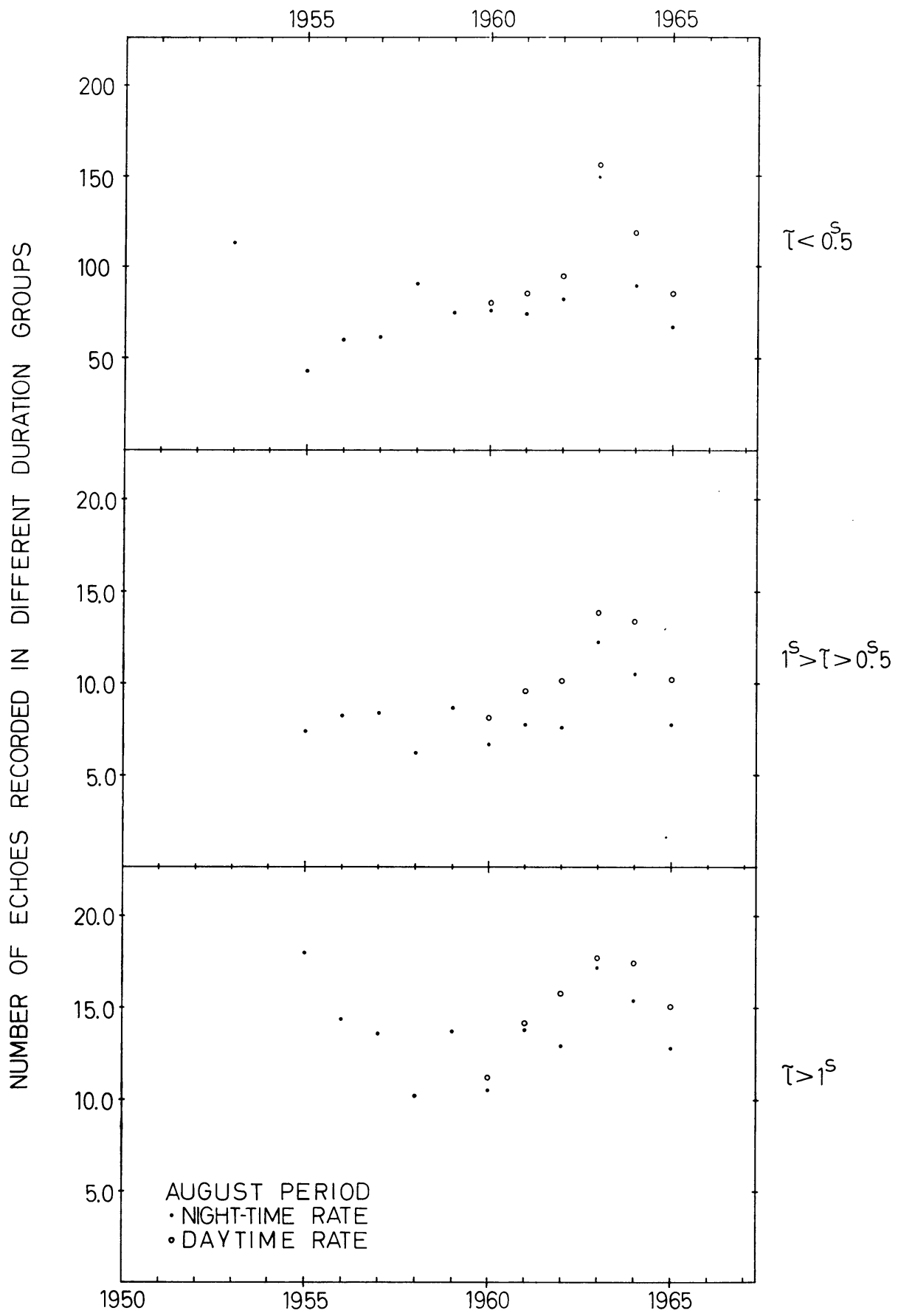

FIG. 2. Night-time and day-time radar hourly rates, August observations 1953-65. Different echoduration groups as indicated. 


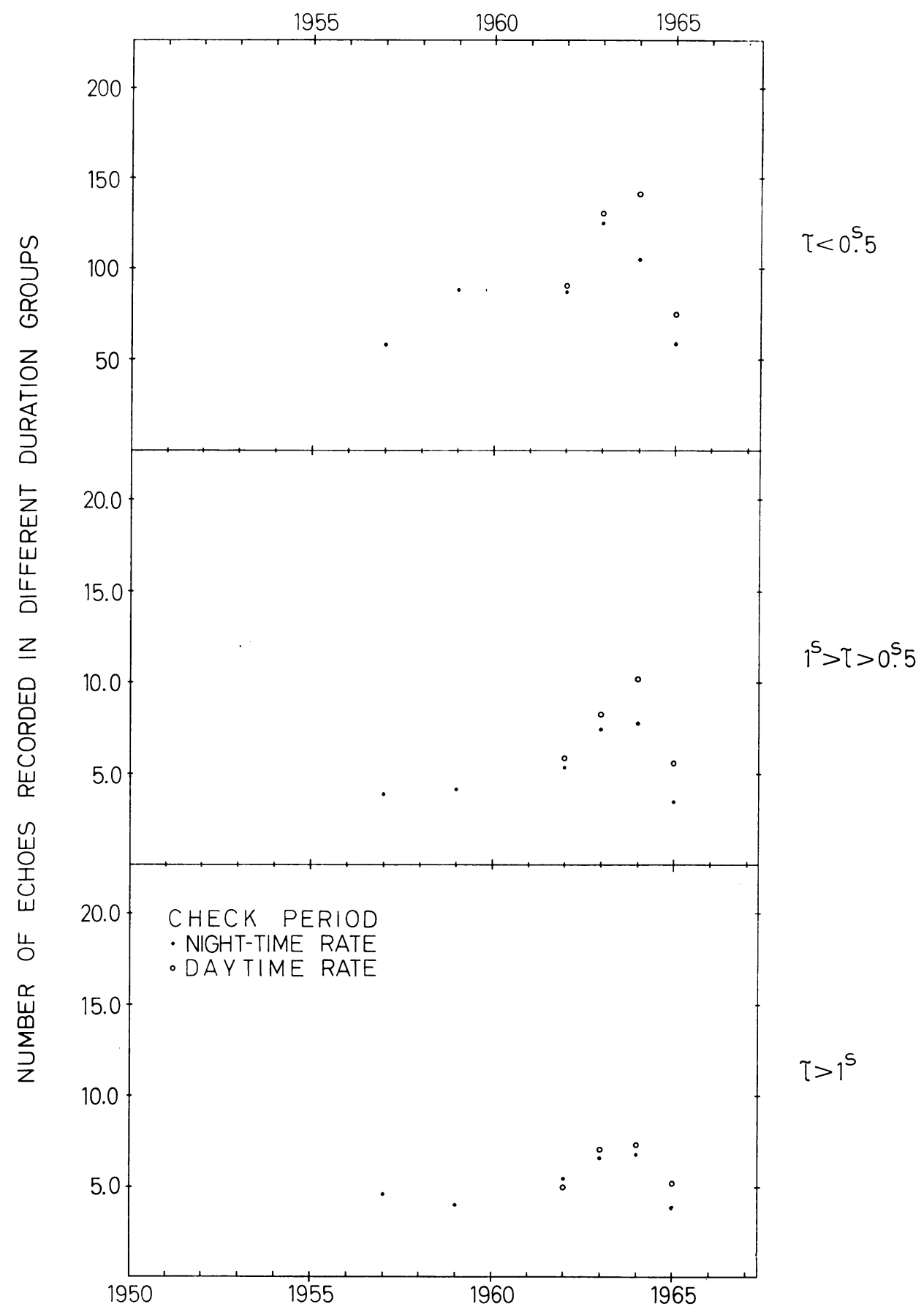

FIG. 3. Night-time and day-time radar hourly rates, control period observations 1957-65. Different echo-duration groups as indicated. 
longer or shorter than $0.5 \mathrm{sec}$. In 1955 the performance of the range-time display deteriorated noticeably and it was not possible to obtain reliable counts of short duration echoes. The 1955 datum points for short-duration echoes are therefore known to be low. A new display unit incorporating both range-time and amplitude-time displays was put into operation in 1956.

Hourly rates for the control periods are plotted in Figure 3. The same timedependent behaviour of echo rates is again evident. The maximum in echo rates, however, appears to have shifted somewhat towards 1964, thus coinciding more closely with the actual solar minimum. The maximum observed echo rate during the control periods exceeds the minimum by a factor of about 2 in the two short-duration data samples, while this factor is slightly less than 2 in the long-duration group.

A comparison of Figures 2 and 3 indicates that the hourly rates of transient echoes are about the same for the August and control periods, while echoes of long duration are more frequent during the August period. The higher percentage of long-duration echoes in August is expected and is explained by the activity of the Perseid shower in mid-August.

In conclusion it may be said that our investigation has not detected any pronounced difference in temporal echo rate variation between short-duration and long-duration meteor echoes. Thus, the statement sometimes made that the 1963 anomalous increase in radar rates was detected only amongst short-duration echoes, and therefore could be a spurious result produced by variations in receiver sensitivity or noise background, appears unfounded.

\section{Mean Atmospheric Penetration Depth}

If, as proposed in Paper I, the observed long-term variations in meteor echo rates are produced by variations in air density at the meteor ionization level, one would expect to detect a corresponding variation in meteor heights and/or meteor trail lengths. An analysis of radar-visual heights (Paper I) indicated that the beginning height, $h_{A}$, exhibited no pronounced variation with time, while the end-point height, $h_{D}$, varied systematically over the time period of the study. The end-point heights of Perseid meteors varied from a lowest value of $85 \mathrm{~km}$ near solar maximum to a highest value of $96 \mathrm{~km}$ near solar minimum. The observed variation in end-heights over the solar cycle is equivalent to a variation in mean Perseid trail length by a factor of $1 \cdot 7$.

For sporadic meteors the angle of incidence is unknown. It is therefore not possible to compute individual trail lengths for sporadic meteors by the radar-visual method. However, if a similar distribution of arrival angles in the annual data samples is assumed, the penetration depth in the atmosphere, $h_{A}-h_{D}$, may be used instead of trail length. 


\section{A. PRESENTATION AND DISCUSSION OF DATA}

The mean penetration depth, $h_{A}-h_{D}$, as recorded in 1953-66, is shown in Figure 4a. Separate curves are given for Perseid and sporadic meteors. The same trend is evident in both curves, with the greatest penetration depths in 1955 and the smallest in 1964 . The absence of radar-visual height data for 1954 and 1957 does not appear to detract from these conclusions. The large scatter evident in the sporadic meteor group is not surprising in view of the extremely small data sample available for study.

The observational material analysed in Figure 4a represents a radar-visual selection of meteors, and the question arises if some peculiarity of this selection could produce the observed secular variation. The two main factors governing the radar-visual

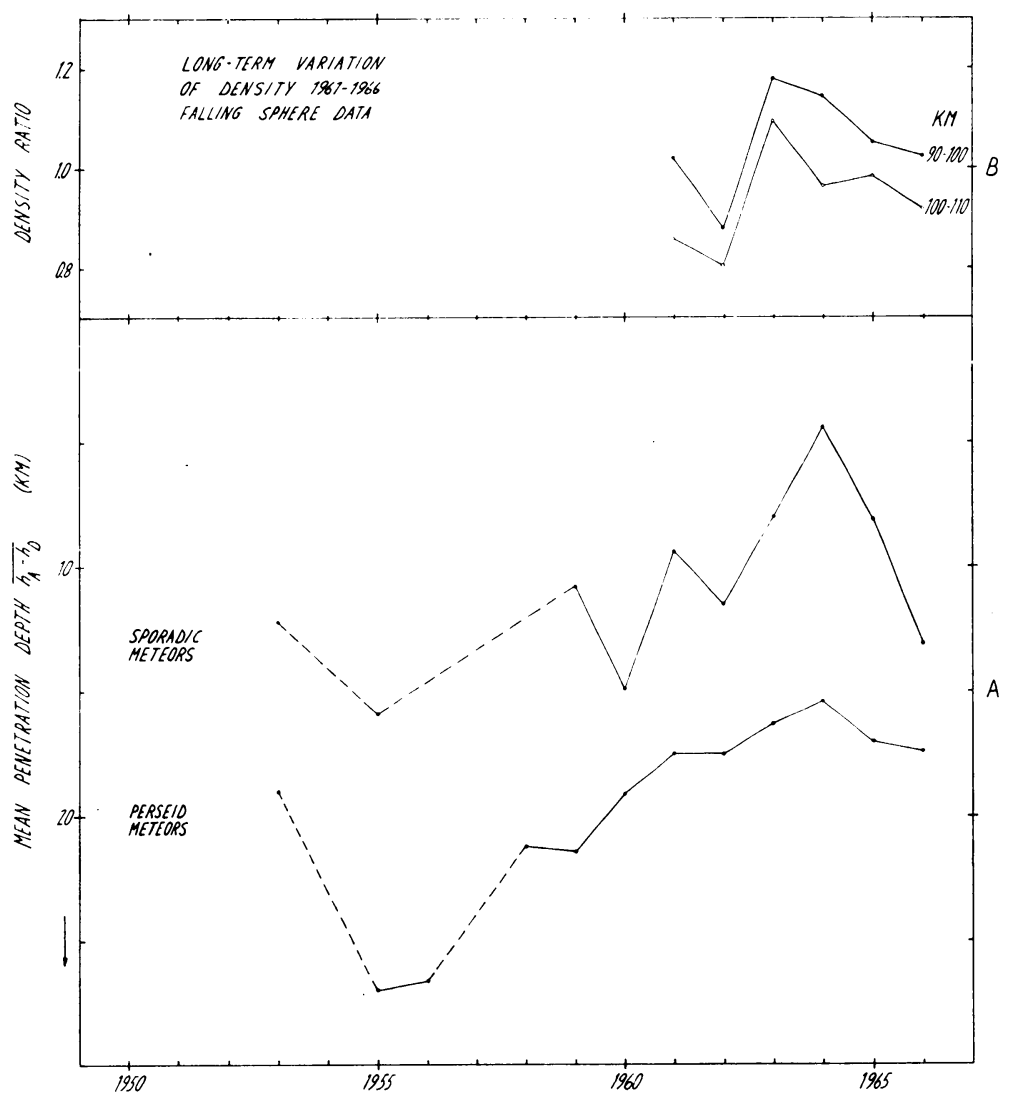

Fig. 4. A. Mean penetration depth $h_{A}-h_{D}$ for Perseid and sporadic meteors as deduced from radar-visual observations 1953-66. - B. Atmospheric density variations 1961-66 as deduced from falling-sphere experiments. Density is given as density ratio, observed density to U.S. 1962 Standard Atmosphere density. 
selection are meteor mass and trail orientation with respect to the radar beam. It appears difficult to imagine any variation in these factors capable of producing the observed time variations in our study.

The diagram, Figure 4, may be compared with a similar diagram obtained by Bumba (1955) from an analysis of visual meteor data. Figure 1 of Bumba's paper indicates that maximum penetration depths and trail lengths of sporadic meteors are recorded 3-4 years after the solar minimum, i.e. near the solar maximum phase. This conclusion is consistent with the results of our study. In addition a second, but smaller, maximum appears in Bumba's data 9-10 years after a solar minimum. The data sample used in Bumba's study represented a compilation from various sources with data collection extending over several solar cycles. The variable length of individual sunspot cycles introduces considerable uncertainty in the analysis. The secondary maxima in penetration depth and trail length found by Bumba 9-10 years after a solar minimum are therefore of doubtful significance.

From the data presented in Figure $4 \mathrm{a}$ of the present paper it is concluded that the longest luminous trails are recorded during - or slightly before - the solar maximum phase, and the shortest meteor trails during solar minimum conditions. This result implies that the typical meteor encounters a steeper atmospheric density gradient at solar minimum than at solar maximum.

\section{Radar-Echo Amplitudes}

Additional evidence for a solar control of the meteoric environment is provided by the analysis of radar-echo amplitudes. The radar receiver used in our study presents meteor echo signals on both range-time and amplitude-time displays. The displays are simultaneously photographed at high film speed. This arrangement, which has been used regularly since 1959 , permits accurate measurements of echo amplitudes.

Echo amplitudes for the 1953-58 period were mainly obtained by direct visual inspection of an A-scope display. The amplitude data of this period are therefore less accurately measured. In the subsequent analysis it was found that a substantial fraction of the weaker echo signals had not been noticed by the A-scope observer although the echo signals were detected on the range-time film. Therefore no amplitude data before 1959 are discussed in the present paper.

Photographic echo amplitudes are available for the 1959-66 period. At present echo amplitudes have been completely reduced only for those echoes which were associated with a simultaneously recorded visual meteor. The reduction procedure involves corrections to observed echo power for radar beam and range (according to an $R^{3}$-power law) as well as minor corrections for variations in transmitter power output (Lindblad, 1956). The echo amplitude measured is the maximum echo amplitude during the first ten echo pulses. The data sample studied includes both specular and non-specular meteor echoes. 


\section{A. PRESENTATION AND INTERPRETATION OF AMPLITUDE DATA}

Mean log reduced echo amplitudes for the 1959-66 period are shown in Figure 5. Separate curves are drawn for Perseid and sporadic meteors. It is seen that both data samples exhibit similar temporal variations, with maximum night-time echo amplitudes being recorded during solar minimum conditions.

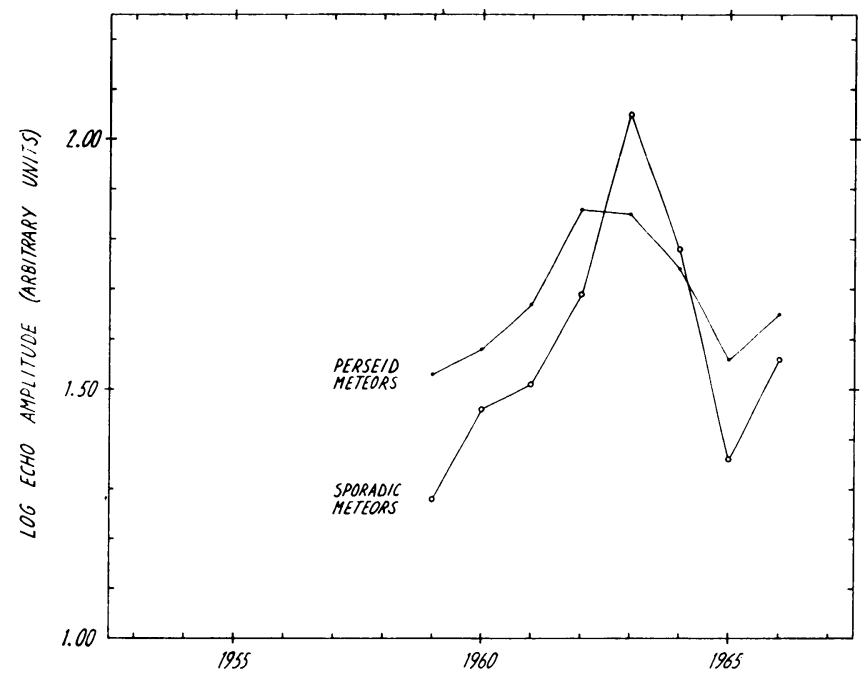

FIG. 5. Mean log reduced echo amplitudes of Perseid and sporadic meteors 1959-66.

In view of the well-known relations: $A \propto \alpha^{1 / 4}$ and $A \propto \alpha$ between echo amplitude $A$ and electron line density $\alpha$, valid for overdense and underdense ionization trails respectively, we may interpret the above result as indicating that the shorter trail lengths observed during minimum solar activity are associated with higher than normal electron line densities. This result is consistent with the previously analysed meteor rate data, which also demand an increase in the echo amplitude and electron line density in order to explain the increase in rates at solar minimum.

The data of Figure 5 represent a radar-visual selection of meteors, and this selection is not independent of echo amplitude nor of noise background. Some caution should therefore be exercised in quantitative interpretation of the echo-amplitude data of Figure 5. The pronounced maximum in echo amplitudes at solar minimum is what one would expect if meteor ionization trails are shortened as the result of a steeper than normal density gradient in the meteor burn-out region. However, the recorded variation in mean echo amplitude, about a factor of 3 over the period of our study, appears unexpectedly large.

The question arises if the observed temporal variation in log echo amplitude could 
to some extent be caused by our calibration procedure. The photographic amplitudetime display is calibrated with the help of an artificial meteor echo signal produced by a reference source. After passing through an automatic step-attenuator the calibration signal is fed capacitively to the receiver input. Echo signal power is measured on the film as a certain number of decibels above the display noise level. At the operating frequency of $32.6 \mathrm{Mc} / \mathrm{s}$, galactic noise far outweighs atmospheric and receiver noise as the factor determining the display noise level. The mean galactic noise level for a given year thus serves as the reference level for amplitude calibration. If long-term variations in absorption of $32 \mathrm{Mc} / \mathrm{s}$ galactic radiation occur in the D-region of the atmosphere, these variations could introduce a spurious time variation in the reduced echo amplitudes. However, the signal path does not pass through the auroral zone and night-time D-region absorption of $32 \mathrm{Mc} / \mathrm{s}$ radiation is, according to present knowledge, negligible during all phases of solar activity. Since it is difficult to conceive of any other effect capable of producing a spurious variation in night-time echo amplitudes, we conclude that the data of Figure 5 are significant.

\section{B. PERCENTAGE OF RADAR-VISUAL ASSOCIATIONS}

An arbitrary index of echo power was obtained by determining the percentage of visual meteors which were detected on the range-time display. The study indicated that the highest percentages of radar-visual coincidences (about 50\%) were observed in 1953 and 1963, and lowest percentage in 1955 and 1956 (about 30\%). The beamed sky area and the area covered by the visual observers was the same throughout the survey. It follows that for given sky area and visual limiting meteor magnitude, more radar echoes are detected above the receiver noise level at solar minimum than at solar maximum. We interpret this result to indicate highest echo amplitudes at solar minimum.

Inspection of the visual magnitudes as recorded by our observing team does not point to any large variation in mean meteor brightness over the solar cycle. This result appears surprising, but it may be explained if one assumes that the magnitude estimates of visual observers refer to the integrated light of a meteor and not to the flux.

\section{Discussion and Concluding Remarks}

The long-term changes in meteor radar rates, atmospheric penetration depths and radar echo amplitudes reported above are all explained qualitatively in terms of a variation in atmospheric density at the meteor burn-out level. It is postulated that in a localized region of the atmosphere, from some 90 to $110 \mathrm{~km}$, neutral density exhibits a long-term variation which is related to position in the solar cycle. Maximum density in this region is assumed to occur during the solar minimum phase. Accordingly, meteor trails and ionization trails are shorter and electron line densities 
are higher at solar minimum than at solar maximum. It follows that for given meteor mass, echo signal strength is increased at solar minimum and more echoes are detected above the radar equipment threshold.

\section{A. COMPARISON WITH ROCKET DATA}

The density variation postulated in the above interpretation of the meteor data has been verified by a direct inspection of rocket-determined densities. In Figure $4 \mathrm{~b}$ are plotted density data for the mesopause and lower thermosphere region, as obtained in 1961-66 from American falling-sphere experiments (Lindblad, 1967b). The density data of Figure $4 \mathrm{~b}$, which all refer to the Northern hemisphere, are given as the ratio observed density to U.S. 1962 Standard Atmosphere density. A density ratio 0.8 thus means that the mean density for a given year, as obtained from all falling-sphere experiments published for that year, was $80 \%$ of the Standard Atmosphere value. Inspection of Figure 4 shows that the long-term changes in rocket and meteor data are remarkably similar.

\section{B. COMPARISON WITH SATELLITE DRAG DATA}

An interesting feature of the present study is that the observed long-term variation in air density at the $90-110 \mathrm{~km}$ level is opposite in phase to the density variation at higher altitudes, as determined from satellite drag studies. A vertical compensation feature is thus observed in the atmosphere, i.e. low densities below $120 \mathrm{~km}$ are associated with high densities above $200 \mathrm{~km}$ during solar maximum activity.

The region of the Earth's atmosphere, from some 120 to $200 \mathrm{~km}$, is the region in which a considerable part of the solar X-ray and EUV-radiation that heats the upper atmosphere is absorbed. One may therefore assume that increased heating of these layers during the solar maximum phase results in an expansion and ascent of an air mass, thus qualitatively explaining the observed increase in air density at satellite altitudes. The air mass which participates in this upward motion is extracted from an atmospheric region below the absorbing layers, and our study indicates that this region may be identified with the meteor burn-out region. Because of the high density in this region, modest density changes can compensate the large density changes at satellite altitudes.

\section{Acknowledgements}

The author is indebted to the director of the Onsala Observatory Professor O. Rydbeck, for his constant interest in this study. Financial support was received from the Swedish Natural Science Research Council. 


\section{References}

Bumba, V. (1949) Bull. astr. Inst. Csl., 1, 93.

Bumba, V. (1955) Bull. astr. Inst. Csl., 6, 139.

Ellyett, C.D., Keay, C.S.L. (1964) Science, 146, 1458.

Lindblad, B.A. (1956) Medd. Lund Obs., Ser. 1, 189.

Lindblad, B. A. (1963) Smithson. Contr. Astrophys., 7, 27.

Lindblad, B.A. (1967a) Space Res., 7, 1028.

Lindblad, B.A. (1967b) Space Res., 8 (in press).

McIntosh, B.A. (1967) Smithson. Contr. Astrophys., 11, 201.

McIntosh, B.A., Millman, P.M. (1964) Science, 146, 1457.

\section{DISCUSSION}

Southworth: You are really talking of a change in the gradient of density, aren't you, rather than a simple change in density. Your results imply a very large change in temperature at this level - some hundreds of degrees.

Lindblad: It is a variation in the density gradient which is observed. Temperature changes occur at some level in the atmosphere, but the height at which these changes occur appears to be different from that of the corresponding changes in density gradient.

Cook: The data presented on end heights imply that the pressure is down at solar maximum and up at solar minimum. The change in radar counts implies longer trails, larger scale height and high temperature at solar maximum. The density of air at satellite height varies in opposition to this, i.e., there is less but warmer air above the meteors at sunspot maximum than at sunspot minimum. It follows that it must on the average be cooler below meteor heights at sunspot maximum and warmer there at sunspot minimum. Also there must be a positive deviation of temperature gradient at sunspot maximum and negative deviation at sunspot minimum, with zero deviation in temperature lying below the height of meteors.

Babadžanov: What is the error in determination of the mean altitude of the disappearance of meteors for the 'altitude-solar activity' diagram?

Lindblad: The error (standard deviation) of one radar-visual height determination is about $3 \mathrm{~km}$. In a mean of 100 meteors the error thus is about $0.3 \mathrm{~km}$.

Bronsten: The solar cycle variations of meteor heights mentioned by Lindblad are in connection with similar variations of the heights of appearance and disappearance of the forbidden green line $\lambda 5577 \AA$ in meteor spectra, investigated by Rajchl in 1964 . It is very interesting to calculate numerically the influence on the solar-cycle effect in the atmosphere by different kinds of high-energy radiations, $\mathrm{X}$-rays, corpuscular and UV radiation.

Fedynskij: Two facts observed by Soviet meteor investigators have confirmed the results obtained by Dr. Lindblad:

(1) Variation of the value

$$
R=\frac{N_{1}}{N_{2}}=\left(\frac{H_{1}}{H_{2}}\right)^{2},
$$

where $R$ is the ratio of numbers of beginning $\left(N_{1}\right)$ and end $\left(N_{2}\right)$ points of the meteor paths, observed in the field of a telescope. This ratio is proportional to the square of the ratio of mean values of heights of the points mentioned above. The value $R$ is diminished for the time of solar activity minimum; this is in a good agreement with the increase of $H_{2}$ at this time.

(2) The percentage of bright meteors which are followed by persistent trains is larger for the time of solar activity minimum. Short papers on both these effects have been published by Astapovič and others.

Lindblad: Several papers on the relation between persistent trains and solar activity have also been published by Czechoslovak astronomers. Their results are, in general, confirmed by our observations. 